\title{
Molecular Sensing Efficiency of Gold-Silver Alloy Nanowires
}

\author{
Weon-Sik Chae, ${ }^{*}$ Hee-Ok Lee, Eun-Mee Kim, and Hyunung Yu ${ }^{\dagger}$ \\ Gangneung Center, Korea Basic Science Institute (KBSI), Gangneung 210-702, Korea. "E-mail: wschae@kbsi.re.kr \\ ${ }^{\dagger}$ Korea Research Institute of Standards and Science (KRISS), Daejeon 305-340, Korea \\ Received July 12, 2010, Accepted February 7, 2011
}

Key Words : Gold-Silver, Alloy, Nanowire, SERS, AAO

Noble metal nanomaterials with a controlled dimension and shape have attracted much attention for their unique physical and chemical properties such as tunable surface plasmonics, surface enhanced Raman scattering (SERS), high-efficiency electrochemical sensing, enhanced fluorescence, and quantum conductance for the last decade. ${ }^{1}$ Surface plasmon resonance characteristics are highly affected by size, shape, and morphology of noble metal nanomaterials. ${ }^{2}$ In the case of silver (Ag) nanomaterials, plasmonic resonance absorption is typically observed in the wide spectral range from ultraviolet (for nanoparticles) to near-infrared (for high aspect ratio nanowires). ${ }^{3}$ Gold ( $\mathrm{Au}$ ) nanomaterials also have tailorable plasmonic characteristics, and there are facile accessible routes to synthesize well-featured nanomaterials. ${ }^{4}$ Recently, porous gold ( $\mathrm{p}-\mathrm{Au}$ ) materials have been developed in different morphology and pore size in order to use them as good SERS substrates because nanopores act as SERS 'hot spot'. Porous gold materials are rationally synthesized through electrochemical gold-silver alloying and silver dealloying route. If the alloying-dealloying route processes in a cylindrical nanopore (e.g., in anodic aluminum oxide membrane), p-Au has a one-dimensional (1D) morphology (i.e., nanowire). ${ }^{6}$

Meanwhile, the composite form of gold-silver (AuAg) alloy has also been synthesized in the different morphologies of particle, wire, and cage in nanometer size. ${ }^{7}$ An alloy nanoparticle supported in a mesoporous matrix has been used as a catalyst for carbon monoxide oxidation. ${ }^{8}$ Unusual electron dynamics is observed in alloy nanoparticles due to a nonequilibrium electron distribution..$^{9}$ A recent notable report is that $\mathrm{AuAg}$ alloy nanocage shows bright three-photon luminescence which enables ultrasensitive and high contrast imaging for biological samples (e.g., cells, tissues, etc.). ${ }^{10}$ Even though AuAg alloy nanomaterials have unique characteristics, there is still a limited study on molecular sensing application using them. ${ }^{11}$

In this study, we prepared AuAg alloy nanowires (NWs) through templating route using anodic aluminum oxide (AAO) templates, and used them as SERS platforms. AuAg alloy was electrochemically deposited in nanocylindrical nanopores, and then the AAO hard template was completely removed by a HF aqueous solution. The resulting alloy nanowires are dispersed in a cresyl violet acetate $(\mathrm{CV})$ solution $\left(1 \times 10^{-6} \mathrm{M}\right)$ under sonication. Raman spectrum was
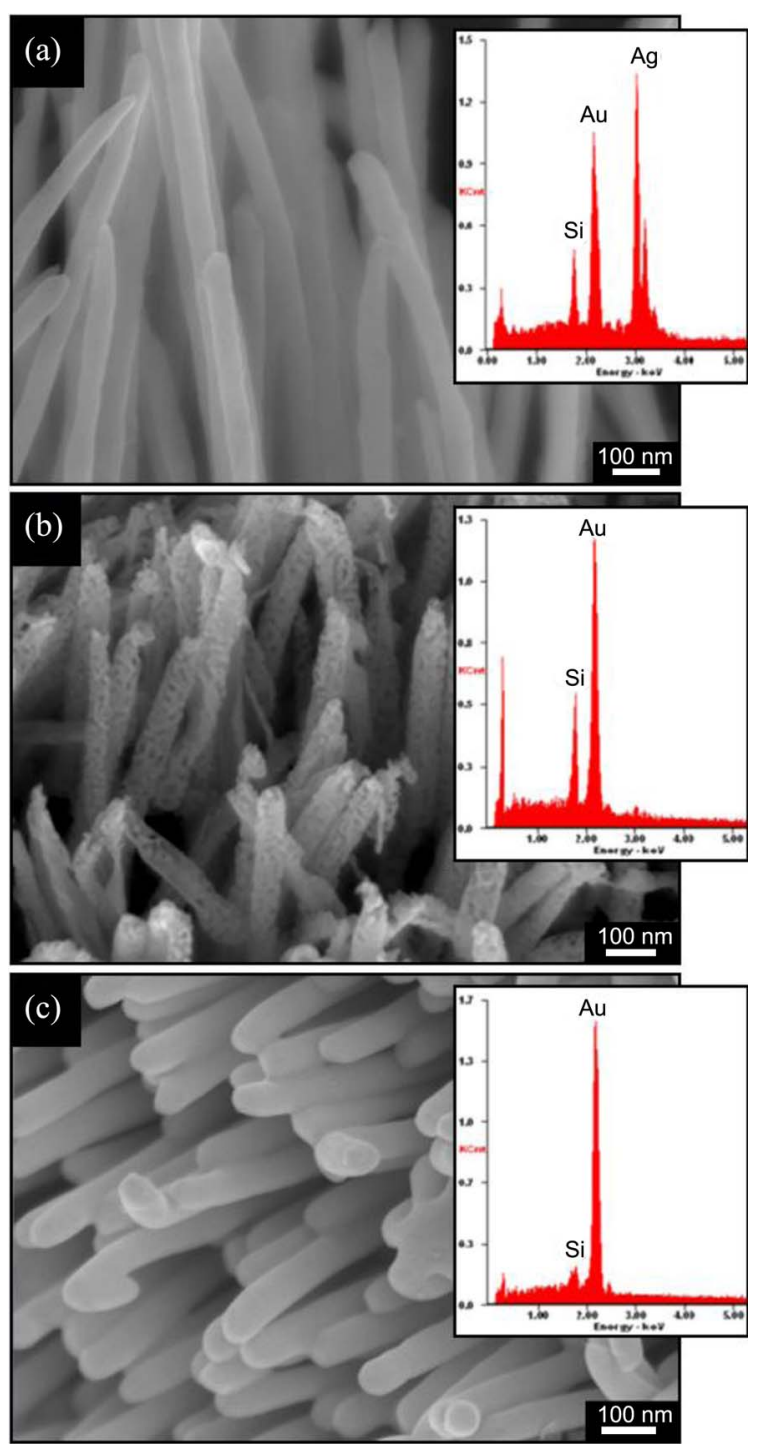

Figure 1. SEM images and EDX spectra (insets) of the AuAg alloy (a), p-Au (b), and s-Au (c) nanowires. The samples are dispersed on silicon wafers for the measurements. 


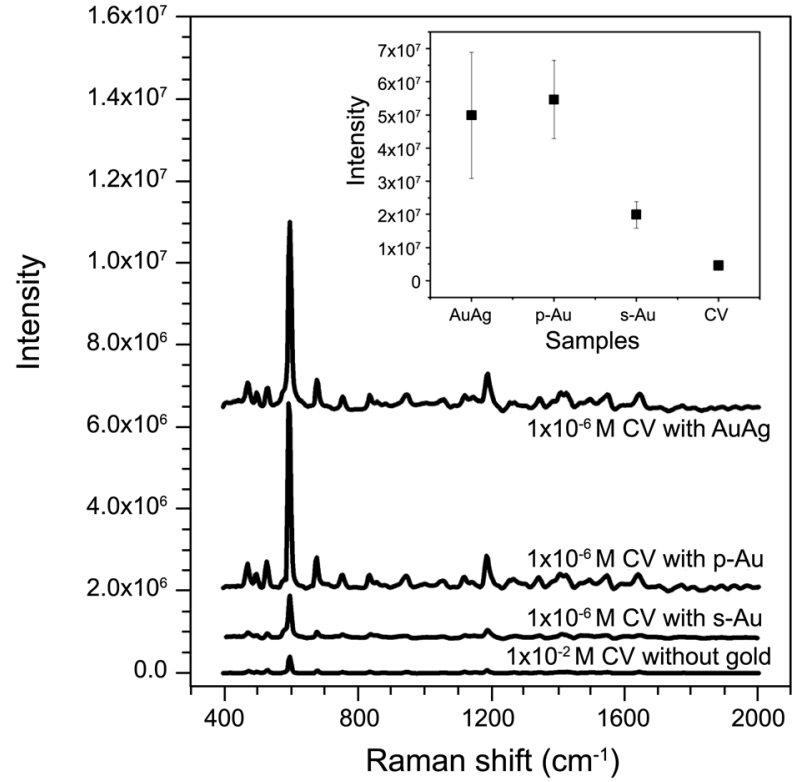

Figure 2. Raman spectra for the $\mathrm{CV}$ probe molecules with the gold nanomaterials; AuAg alloy, p-Au, and s-Au NWs. Inset is the SERS intensity measured at $592 \mathrm{~cm}^{-1}$ with an error range for a better comparison.

measured on a home-built micro-Raman microscope using a He-Ne laser $\left(\lambda_{\text {ex }}=633 \mathrm{~nm}\right)$.

The resulting template-free alloy, $\mathrm{p}-\mathrm{Au}$, and solid $\mathrm{Au}$ without pores $(\mathrm{s}-\mathrm{Au})$ have 1D wire morphology with an average diameter of $\sim 55 \mathrm{~nm}$ (Fig. 1) which is well correlated with the inner pore size of the AAO templates. The p-Au NWs show a porous network having a pore size of $\sim 10 \mathrm{~nm}$ (Fig. 1(b)). Energy-dispersive X-ray (EDX) spectra reveal that the AuAg alloy NWs have an average atomic composition of $\mathrm{Au}_{24} \mathrm{Ag}_{76}$, while the $\mathrm{p}-\mathrm{Au}$ and the s-Au NWs consist of pure gold atoms.

Figure 2 shows the representative Raman spectra of CV probe solution including the gold nanomaterials. The observed Raman signal intensity is dramatically enhanced for the CV with the gold nanomaterials compared with that of the bulk CV solution without gold. Enhancement factor $(\mathrm{EF})$, which is an intensity ratio $\left(\mathrm{I}_{\mathrm{SERS}} / \mathrm{I}_{\mathrm{RS}}\right)$ of an observed SERS for molecules with a metal to a normal Raman scattering without a metal, is estimated by comparing the peak at $592 \mathrm{~cm}^{-1}$. This peak corresponds to the characteristic $\mathrm{C}-\mathrm{N}-\mathrm{C}$ and C-C-C in-plane skeletal deformation modes of the $\mathrm{CV}$ probe molecule. The estimated $\mathrm{EF}$ values are summarized in Table 1. For all the gold nanomaterials examined, the $\mathrm{EF}$ values are above $\sim 10^{5}$. Furthermore, it is notable to mention that $\mathrm{I}_{\mathrm{SERS}}$ for the $\mathrm{CV}$ probe is stronger with the AuAg alloy and the p-Au NWs than with the s-Au NWs. The AuAg alloy NWs show a comparable SERS activity to the p-Au NWs. It is previously reported that the strong SERS activity with the p-Au NWs is attributed to nanopores, which work as 'hot spot', as well as enlarged surface area. ${ }^{6 c}$

Previously, plasmonic nanomaterials with characteristic features, such as nanopore, roughened surface, and bimetallic composition, typically showed intense SERS signals. ${ }^{6 c, 12,13}$
Table 1. EF values of the gold nanowires examined for SERS experiments

\begin{tabular}{ccc}
\hline Samples & EF value & $\mathrm{EF}_{\text {sample }} / \mathrm{EF}_{\text {s-Au }}$ \\
\hline AuAg alloy NW & $\sim 1.1 \times 10^{6}$ & $\sim 8.5$ \\
p-Au NW & $\sim 1.2 \times 10^{6}$ & $\sim 9.2$ \\
s-Au NW & $\sim 1.3 \times 10^{5}$ & - \\
\hline
\end{tabular}

Here, the AuAg alloy NWs does not have any distinct features of nanopore and roughened surface compared to the s-Au NWs (Fig. 1). Meanwhile, it was previously reported that uneven elemental distribution could be formed in bimetallic nanomaterials, for examples, solid solution, coreshell, and multilayer structure. ${ }^{12,13}$ Notable SERS activity was observed in such the bimetallic nanomaterials, which could be explained by local electromagnetic fields caused by plasmon coupling between constituent phases. ${ }^{13}$

In this study, the fabricated AuAg alloy NW is a solid solution consisting of gold and silver elements. According to a previous report, ${ }^{14}$ electroplated bulk AuAg alloy has homogeneous mixture of component elements. However, it may not be real in the electrochemically grown alloy NWs inside AAO nanocylinders since the hydrophilic alumina surface seems to be more attractive to one of the constituent elements. To prove plausible spatial disproportionation of elements, supplementary SERS experiment is performed using pyridine in this study, as a standard probe molecule, ${ }^{11}$ and it is clearly proved that $\mathrm{Ag}$ atom is rich at the surface of the alloy NWs (data not shown), which means that Ag atom has higher affinity to the alumina template during electroplating. Hence, it is considered that the uneven distribution of $\mathrm{Au}$ and $\mathrm{Ag}$ (surface rich) elements seems to be a reason for the observed intense SERS signal when using the AuAg alloy NW platform through plausible plasmon coupling, in addition to minor chemical effects between the alloy and the probe molecules.

In summary, we estimated SERS efficiency for the AuAg alloy NWs which were prepared by electrochemical alloying using AAO hard templates. The estimated SERS efficiency for the alloy NWs is comparable to the p-Au NWs, and both the NWs show definitely higher molecular sensing activity than the s-Au NWs. This result means that 1D AuAg alloy nanomaterials would be excellent SERS substrates.

\section{Experimental Section}

Preparation of AuAg Alloy Nanowires. Firstly AuAg alloy was electrochemically deposited in the nanocylindrical pores of the AAO membrane. The AAO membrane was Pt back-plated using a sputter to use as a working electrode prior to the alloy deposition. A two-electrode cell configuration with a Pt counter electrode was used for the electrochemical deposition using a $20 \mathrm{~mL}$ aqueous solution consisting of $0.05 \mathrm{M} \mathrm{KAg}(\mathrm{CN})_{2}$ and $0.25 \mathrm{M} \mathrm{Na}_{2} \mathrm{CO}_{3}$ at $2.7 \mathrm{~V}$ for $30 \mathrm{~s}$ for Ag buffer layer and in a $20 \mathrm{~mL}$ solution consisting of $0.02 \mathrm{M} \mathrm{KAu}(\mathrm{CN})_{2}, 0.05 \mathrm{M} \mathrm{KAg}(\mathrm{CN})_{2}$ and $0.25 \mathrm{M}$ $\mathrm{Na}_{2} \mathrm{CO}_{3}$ at $2.7 \mathrm{~V}$ for $10 \mathrm{~min}$ for the AuAg alloy in constant 
potential mode. The AAO template was completely removed by a $10 \mathrm{wt} \% \mathrm{HF}$ aqueous solution, which resulted in $\mathrm{AuAg}$ alloy nanowires. Silver atoms of the AuAg alloy were selectively etched out within $20 \mathrm{~min}$ in concentrated nitric acid, leaving porous $\mathrm{Au}$ nanowires. For the comparative SERS study, smooth solid Au nanofibers were also synthesized using the electrolyte including $0.02 \mathrm{M} \mathrm{KAu}(\mathrm{CN})_{2}$ and 0.25 $\mathrm{M} \mathrm{Na}_{2} \mathrm{CO}_{3}$ at $2.7 \mathrm{~V}$ for $10 \mathrm{~min}$.

Instrumentations. Scanning electron microscope (SEM) imaging was carried on a microscope (Hitachi, SU-70) equipped with an EDX spectrometer. Raman spectra were measured on a home-built micro-Raman setup with an inverted microscope (Zeiss, Axiovert-40CFL). A He-Ne laser $\left(\lambda_{\text {ex }}=633 \mathrm{~nm}, 5 \mathrm{~mW}\right)$ and a TE-cooled CCD (Princeton Instruments, PIXIS: 100B) detector were used to collect the Raman scattered light from the CV probe molecules after removal of Rayleigh scattering. SERS spectra were obtained at multiple locations for each solution sample including $\mathrm{CV}$ probes $\left(1 \times 10^{-6} \mathrm{M}\right)$ and the $1 \mathrm{D}$ gold and gold-silver nanomaterials with an integration time of $100 \mathrm{~s}$ for all measurements. SERS experiment was also examined for pyridine $\left(1 \times 10^{-2} \mathrm{M}\right)$ solution without gold nanomaterials.

Acknowledgments. This work was supported by International Joint Research Program (F31603) from KBSI. H.Y. acknowledges partial support of Nano R\&D program through the Korea Science and Engineering Foundation funded by the Ministry of Education, Science and Technology (200804406).

\section{References}

1. (a) Tao, A.; Sinsermsuksakul, P.; Yang, P. Nature Nanotech. 2007, 2, 435. (b) Campion, A.; Kambhampati, P. Chem. Soc. Rev. 1998, 27, 241. (c) Kneipp, K.; Kneipp, H.; Kneipp, J. Acc. Chem. Res. 2006, 39, 443. (d) Yoon, K. J.; Seo, H. K.; Hwang, H.; Pyo, D.; Eom, I.-Y.; Hahn, J. H.; Jung, Y. M. Bull. Korean Chem. Soc. 2010, 31, 1215. (e) Liu, Z.; Searson, P. C. J. Phys. Chem. B 2006, 110, 4318. (f) Zhang, Y.; Dragan, A.; Geddes, C. D. J. Phys. Chem. C 2009, 113, 12095. (g) Kondo, Y.; Takayanagi, K. Science 2000, 289, 606. (h) Rodrigues, V.; Fuhrer, T.; Ugarte, D. Phys. Rev. Lett. 2000, 85, 4124.

2. (a) Emory, S. R.; Haskins, W. E.; Nie, S. J. Am. Chem. Soc. 1998, 120, 8009. (b) Seney, C. S.; Gutzman, B. M.; Goddard, R. H. J. Phys. Chem. C 2009, 113, 74. (c) Noguez, C. J. Phys. Chem. C
2007, 111, 3806. (d) Tiwari, V. S.; Oleg, T.; Darbha, G. K.; Hardy, W.; Singh, J. P.; Ray, P. C. Chem. Phys. Lett. 2007, 446, 77. (e) Jeong, D. H.; Zhang, Y. X.; Moskovits, M. J. Phys. Chem. B 2004, 108, 12724. (f) Mohanty, P.; Yoon, I.; Kang, T.; Seo, K.; Varadwaj, K. S. K.; Choi, W.; Park, Q.-H.; Ahn, J. P.; Suh, Y. D.; Ihee, H.; Kim, B. J. Am. Chem. Soc. 2007, 129, 9576. (g) Bae, Y.; Kim, N. H.; Kim, M.; Lee, K. Y.; Han, S. W. J. Am. Chem. Soc. 2008, 130, 5432. (h) Lu, L.; Eychmüller, A. Acc. Chem. Res. 2008, 41, 244. (i) Park, J. S.; Yoon, J. H.; Kim, H. J.; Huh, Y.-D.; Yoon, S. Bull. Korean Chem. Soc. 2010, 31, 819. (j) Brolo, A. G.; Arctander, E.; Gordon, R.; Leathem, B.; Kavanagh, K. L. Nano Lett. 2004, 4, 2015. (k) Hunyadi, S. E.; Murphy, C. J. J. Mater. Chem. 2006, 16, 3929.

3. (a) Mulvaney, P. Langmuir 1996, 12, 788. (b) Evanoff, D. D.; Chumanov, G. J. Phys. Chem. B 2004, 108, 13948. (c) Murphy, C. J.; Jana, N. R. Adv. Mater. 2002, 14, 80. (d) Tao, A.; Kim, F.; Hess, C.; Goldberger, J.; He, R.; Sun, Y.; Xia, Y.; Yang, P. Nano Lett. 2003, 3, 1229 .

4. (a) Gao, J.; Bender, C. M.; Murphy, C. J. Langmuir 2003, 19 9065. (b) Chen, H. M.; Peng, H.-C.; Liu, R.-S.; Asakura, K.; Lee, C.-L.; Lee, J.-F.; Hu, S.-F. J. Phys. Chem. B 2005, 109, 19553.

5. (a) Qian, L. H.; Yan, X. Q.; Fujita, T.; Inoue, A.; Chen, M. W. Appl. Phys. Lett. 2007, 90, 153120. (b) Gao, W.; Xia, X.-H.; Xu, J.-J.; Chen, H.-Y. J. Phys. Chem. C 2007, 111, 12213. (c) Zhang, X.; Li, D.; Bourgeois, L.; Wang, H.; Webley, P. A. Chem. Phys. Chem. 2009, 10, 436.

6. (a) Ji, C.; Searson, P. C. Appl. Phys. Lett. 2002, 81, 4437. (b) Bok, H.-M.; Shuford, K. L.; Kim, S.; Kim, S. K.; Park, S. Nano Lett. 2008, 8, 2265. (c) Lee, H.-O.; Kim, E.-M.; Yu, H.; Jung, J.-S.; Chae, W.-S. Nanotechnol. 2009, 20, 325604.

7. (a) Mallin, M. P.; Murphy, C. J. Nano Lett. 2002, 2, 1235. (b) Ji, C.; Searson, P. C. J. Phys. Chem. B 2003, 107, 4494. (c) Chen, J.; McLellan, J. M.; Siekkinen, A.; Xiong, Y.; Li, Z.-Y.; Xia, Y. J. Am. Chem. Soc. 2006, 128, 14776.

8. Wang, A.; Hsieh, Y.-P.; Chen, Y.-F.; Mou, C.-Y. J. Catal. 2006, 237, 197.

9. Link, S.; Burda, C.; Wang, Z. L.; El-Sayed, M. A. J. Chem. Phys. 1999, 111,1255

10. Tong, L.; Cobley, C. M.; Chen, J.; Xia, Y.; Cheng, J.-X. Angew. Chem. Int. Ed. 2010, 49, 3485.

11. Kim, K.; Kim, K. L.; Lee, S. J. Chem. Phys. Lett. 2005, 403, 77.

12. (a) Lu, L.; Eychmller, A.; Kobayashi, A.; Hirano, Y.; Yoshida, K.; Kikkawa, Y.; Tawa, K.; Ozaki, Y. Langmuir 2006, 22, 2605. (b) Kumar, G. V. P.; Shruthi, S.; Vibha, B.; Reddy, B. A. A.; Kundu, T. K.; Narayana, C. J. Phys. Chem. C 2007, 111, 4388.

13. (a) Hunyadi, S. E.; Murphy, C. J. J. Mater. Chem. 2006, 16, 3929. (b) Huang, Y.; Yang, Y.; Chen, Z.; Li, X.; Nogami, M. J. Mater. Sci. 2008, 43, 5390. (c) Zhu, J. Nanoscale Res. Lett. 2009, 4, 977.

14. Erlebacher, J.; Aziz, M. J.; Karma, A.; Dimitrov, N.; Sieradzki, K. Nature 2001, 410, 450. 